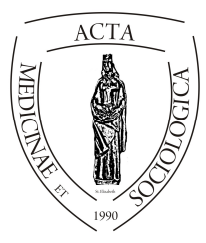

doi: $10.19055 / \mathrm{ams} .2018 .9 / 26 / 6$

\title{
Hungarian students' choices between complementary and alternative medicine or conventional medicine for themselves and family members on a five-level severity scale of different diseases
}

\author{
Andrea Sárváry ${ }^{1}$, Péter Takács ${ }^{2}$, Attila Sárváry $^{3}$ \\ 1. föiskolai docens, Debreceni Egyetem Egészségügyi Kar, Szociális és Társadalomtudományi Intézet, \\ 4400 Nyíregyháza, Sóstói út 2-4. \\ 2. főiskolai tanár, Debreceni Egyetem Egészségügyi Kar, Egészségtudományi Intézet, 4400 Nyíregyháza, Sóstói út 2-4. \\ 3. föiskolai docens, Debreceni Egyetem Egészségügyi Kar, Egészségtudományi Intézet, 4400 Nyíregyháza, Sós- \\ tói út 2-4.
}

\section{INFO}

\section{Andrea Sárváry \\ sarvary.andrea@foh.unideb.hu}

\section{Keywords}

complementary and alternative medicine; conventional medicine; students' choice; family members; severity of diseases

\section{ABSTRACT}

\begin{abstract}
The primary aim of this study was to examine how students choose between complementary and alternative medicine (CAM) and conventional medicine (COM) on a 5point severity scale of diseases and how their choices change as the severity of diseases increases. The study also aimed to reveal whether the students' choices between CAM and COM for themselves and for their family members are consistent. Finally, comparisons were made between male and female students.
\end{abstract}

Methods: In a cross-sectional survey 725 (44.6\% response rate) health care and social work students at the University of Debrecen, Faculty of Health completed the questionnaire. Data were analysed using descriptive and inferential statistics.

Results: On each level of severity most of the students chose 'primarily COM, secondly CAM' (38.8\%-64.5\%), followed by 'only COM' (between $23.5 \%$ and $28.0 \%$ ), CAM+COM (between 9.7\% and 27.0\%), and finally, 'only CAM' (between 2.3\% and 6.0\%).). Students' choices between CAM and COM for themselves and for their family members were consistent. Significantly more males than females chose 'only COM' on the mildest, serious and the most serious levels $(37.6 \%$ vs. $26.7 \%$; $37.9 \%$ vs. $23.9 \%$; $38.2 \%$ vs. $21.4 \%$; $\mathrm{p}<0.05$ ) and significantly more females than males chose the 'primarily COM and secondly CAM' on serious and the most serious levels of disease $(65.4 \%$ vs. $50.8 \%$; $66.4 \%$ vs. $50.8 \%$; p $<0.05)$. The more serious the disease was, the response rate in 'primarily COM and secondly CAM' increased while it decreased in 'primarily 
CAM and secondly COM', in 'only CAM' and in 'only COM'. As the severity of disease increased one part of students changed their choices between CAM and COM.

Conclusions: Further analyses are needed to reveal the patterns of choices between CAM and COM as the severity of disease increases.

Kulcsszavak
komplementer és alterna-
tív medicina,
hagyományos orvoslás,
hallgatók választásai,
családtagok,
betegségek súlyossága

Absztrakt: Kutatásunk egyik célja annak vizsgálata volt, hogy kiderítsük, hogyan választanak a hallgatók a komplementer és alternatív medicina (CAM) és a hagyományos orvoslás között (COM) a betegségek 5 súlyossági szintjén, és választásuk hogyan változik a betegségek súlyosságának erősödésével. Másik célunk annak kiderítése volt, hogy a hallgatók CAM és COM közötti választása saját maguk és családtagjaik számára konzisztens-e. Továbbá, vizsgáltuk a férfi és női hallgatók választása közötti különbségeket.

Módszer: Keresztmetszeti kutatásunkban a Debreceni Egyetem Egészségügyi Karának 725 egészségügyi és szociális munkás hallgatója vett részt (válaszarány: 44.6\%). Az adatokat leíró és inferenciális statisztikai módszerekkel elemeztük.

Eredmények: A betegségek minden súlyossági szintjén a hallgatók többsége az ,elsősorban COM, másodsorban CAM" lehetőséget választották (38.8\% és $64.5 \%$ között), ezt követte a „csak COM” (23.5\% és 28\% között), a „CAM+COM" (9.7\% és $27 \%$ között), és végül a „csak CAM” ( $2.3 \%$ és $6 \%$ között). A hallgatók CAM és COM közötti választása saját maguk és családtagjaik számára konzisztens volt. Szignifikánsan több férfi, mint nő választotta a „,csak COM"-t a legenyhébb, súlyos és legsúlyosabb szinteken (37.6\% vs. $26.7 \% ; 37.9 \%$ vs. $23.9 \% ; 38.2 \%$ vs. $21.4 \%$; $\mathrm{p}<0.05$ ), és szignifikánsan több nő, mint férfi választotta az „elsősorban COM, másodsorban CAM"-t a betegségek súlyos és legsúlyosabb szintjein $(65.4 \%$ vs. $50.8 \%$; $66.4 \%$ vs. $50.8 \% ; \mathrm{p}<0.05)$. A betegség súlyosságának erősödésével a válaszarány növekedett az ,elsősorban COM, másodsorban CAM”, és csökkent az „elsősorban CAM és másodsorban COM”, a „csak COM” és a „csak CAM” kategóriákban. A súlyosság erősödésével a hallgatók egy része megváltoztatta a CAM és COM közötti választását.

Következtetések: További kutatás szükséges a betegségek súlyosságának erősödése során kirajzolódó CAM és COM közötti választási mintázatok kiderítésére.

\section{Introduction}

Complementary and alternative medicine (CAM) is still popular worldwide (Büssing, Ostermann et al. 2011, Fox, Coughlan et al. 2010, Barnes, Bloom and Nahin 2008) as it is reflected in the prevalence data of CAM use that varied between $9.8 \%$ and $76 \%$ 
among general population (Harris, Cooper et al. 2012, Büssing, Ostermann et al. 2011, Fox, Coughlan et al. 2010, Barnes, Bloom and Nahin 2008). The popularity of CAM among patients with chronic illness is similar or even higher than among the average population (Akinci, Zenginet et al. 2011, Akyol and Oz 2011, Molassiotis, FernandezOrtega et al. 2005a). Previous studies indicated that CAM use increases with serious clinical and health conditions (Reid, Steel et al. 2016, Ni, Simile and Hardy 2002) and it is exceptionally high among patients with life threatening illnesses such as cancer (Sparber and Wootton 2001) or HIV (Wootton and Sparber 2001). A lot of studies focused on patients with cancer (John, Hershman et al. 2016, Dhanoa, Yong et al. 2014, Horneber, Bueschel et al. 2012, Molassiotis, Fernandez-Ortega et al. 2005a), surgery patients (Schieman, Rudmik et al. 2009, Norred 2002, Crowe, Fitzpatrick and Jamaluddin 2002, Wang, Peloquin and Kain 2002, Norred, Zamudio and Palmer 2000), cardiac patients (Mandreker 2015) and diabetic patients (Chang, Wallis and Tiralongo 2007). The rate of CAM use by cancer patients was higher among younger patients, females and patients with a higher educational level (Molassiotis, FernadezOrtega et al. 2005b), however among diabetes patients the main determining factors were age, duration of diabetes, degree of complications and self-monitoring of blood glucose (Chang, Wallis and Tiralongo 2007).

Although previous two national representative surveys showed an increased visiting rate of CAM practitioners from $6.6 \%$ to $23.9 \%$ between 1991 and 1999 (23.9\%) (Buda 2003, Antal and Szántó 1992), this rate was found to be 8.9\% only in the latest survey carried out in 2009 (Európai Lakossági Egészségfelmérés, 2011). Our previous study found more than 50\% personal use in herbal medicine, massage and relaxation among health care students (Sárváry, Demcsák et al. 2016). The prevalence of herbal medicine use was $7.2 \%$ among Hungarian patients who had undergone elective surgery (Soós, Jeszenői et al. 2015).

Numerous studies have already been published about the knowledge, perception and attitudes toward CAM among health care students and health professionals (Sárváry, Demcsák et al. 2016, Chang and Chang 2015, Trail-Mahan, Mao and BawelBrinkley 2013, Akan, Izbirak et al. 2012, Awad, Al-Ajmi and Waheedi 2012, Shorofi and Arbon 2010, Fountouki and Theofanidis 2009). These studies revealed a positive attitude towards CAM among health care students and found that female students had a more positive attitude towards CAM therapies and they used CAM more frequently than male students (Sárváry, Demcsák et al. 2016, Akan, Izbirak et al. 2012, Awad, Al-Ajmi and Waheedi 2012, Greenfield, Brown et al 2006).

In contrast to the growing body of knowledge concerning patients' use of CAM, little is known on the choices that students make between CAM and conventional medicine $(\mathrm{COM})$ with consideration for different levels of disease severity. Therefore, the primary aim of this study was to examine how students choose between CAM and COM on a 5-point severity scale of diseases and how their choices change as the severity of diseases increases. The study also aimed to reveal whether the students' choices between CAM and COM for themselves and for their family members are consistent. According to cognitive consistency theories people seek consistency in their thinking (Smith and Mackie 2007), so students' choices may be the same for themselves and for their family members. However, because of the age factor the choice between CAM and COM may be different for the students themselves and for 
their child or parent. As the third aim of the study comparisons were made between male and female students. The framework of this study is presented in Figure 1.

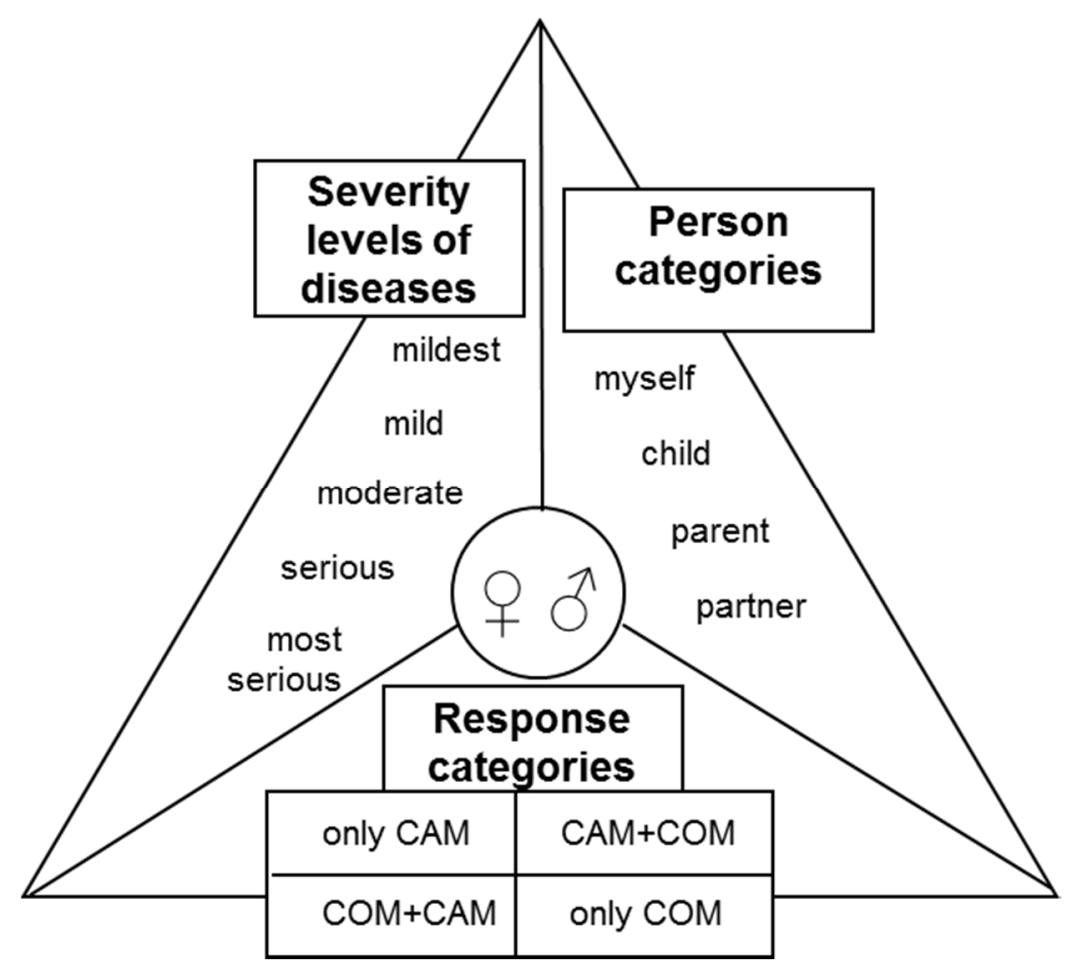

Figure 1 Framework of this study.

\section{Materials and methods}

\section{Study Questionnaire}

In the questionnaire a 5 point severity scale of diseases, four 'person categories' and four response categories were defined and utilized. The 5 point severity scale of diseases included the following levels:

- mildest: diseases lasting for some days only,

- mild: diseases lasting for some weeks without any complications,

- moderate: diseases demanding hospital care,

- serious: chronic diseases, and diseases deteriorating life quality,

- most serious: life-threatening or incurable diseases. 
The person categories were as follows: the respondent him/herself, his/her (future) child, his/her parents and his/her partner.

The response categories were as follows:

- only CAM,

- primarily CAM and secondly conventional medicine (abbreviated as ' $\mathrm{CAM}+\mathrm{COM}$ '),

- only conventional medicine (abbreviated as 'only COM') and

- primarily conventional medicine and secondly CAM (abbreviated as 'COM+CAM').

On each level of severity of diseases respondents were asked to indicate which response categories they would choose for themselves, their (future) child, their parents and their partner in the case of a disease. (E.g. Which response category would you choose if you or one of your relatives had one of the mildest diseases that last for some days only?)

\section{Study sample}

The study sample consisted of $1^{\text {st }}-4^{\text {th }}$-year students from the Faculty of Health of the University of Debrecen. The sample was composed of full time (996) and part time (631) students; a total of 1627, of which 259 were males and 1368 females: 294 nurses, 256 paramedics, 211 midwives, 196 health visitors, 332 health care managers and 338 social workers.

\section{Pilot study}

The questionnaire was pilot-tested for content, easy usage, language clarity, and time required to complete. It was pre-tested with 8 students, and necessary modifications were made so that it would be simple to answer, yet provide accurate data.

\section{Data collection}

The study was carried out at the University of Debrecen, Faculty of Health in Nyíregyháza, Hungary between April and June 2013. The Eva-sys online version of the questionnaire was used in order to increase the efficiency of data collection. Completion of the questionnaire occurred during seminars when computers were available for students. Students had only one occasion to complete the questionnaire. Teachers gave the students a brief description of what the study entailed before they filled in the questionnaire. Participation in the study was voluntary and a written permission to conduct the research was obtained from the University of Debrecen, Faculty of Health.

\section{Statistical analysis}

All the data were analysed using the Statistical Package for Social Sciences (SPSS) software program (Version 22.0). To summarize the data descriptive statistics were used. Analysis of proportion was used to compare the frequencies (Statistical Data Analysis, 2016). Results were considered to be significant when the p-value was less than 0.05 . 


\section{Choice between CAM and COM on the five levels}

Average frequencies were calculated in all the four response categories on the five levels. (e.g. on the mildest level the frequencies of 'myself', 'child', 'parent' and 'partner' in the 'only CAM' response category were added up and divided by 4 .)

Students' choices between CAM and COMfor themselves and for their family members

Analysis of proportion was used to compare the frequencies of the four "person-categories' with each other in the four response categories on each five level. E.g. on the mildest level the frequencies of 'only CAM' were compared between myself and child, between myself and parents, between myself and partner, between child and parent, between child and partner, and between parent and partner.

Changes in the choices between CAM and COM as the severity of diseases increases

Analysis of proportion was used to compare the average frequencies of the four response categories one by one with the subsequent levels of severity.

Gender difference of the choices between CAM and COM on the five levels of severity of diseases

Step 1 Average frequencies were calculated for males and females in the four response categories on the five levels. E.g. on the mildest level the frequencies of the four person-categories in 'only CAM' were added up and divided by 4 in the cases of males. Step 2 Analysis of proportion was used to compare the average frequencies between males and females in the four response categories on each five level. E.g. the average frequency of the only CAM was compared between males and females on the mildest level.)

Gender difference of the choices between CAM and COM as the severity of the diseases increases

Analysis of proportion was used to compare the average frequencies in the four response categories one by one between males and females with the subsequent levels of severity.

\section{Results}

Seven hundred and twenty-five $1^{\text {st }}-4^{\text {th }}$-year students $(93,12.8 \%$ males, and 632 , $87.2 \%$ females) completed the questionnaire (Table 1), giving a response rate of $44.6 \%(725 / 1627)$. The sample was representative with respect to proportions of gender attending the Faculty of Health. Respondents consisted of the following groups: $185(25.5 \%)$ nurses, $62(8.6 \%)$ paramedics, $125(17.2 \%)$ midwives, $99(13.7 \%)$ health 
visitors $^{1}, 104(14.3 \%)$ health care managers and $150(20.7 \%)$ social workers. Data were collected from $1^{\text {st }}$ year students $(289,39.9 \%), 2^{\text {nd }}$ year $(217,29.9 \%), 3^{\text {rd }}$ year $(142,19.6 \%)$, and $4^{\text {th }}$ year $(77,10.6 \%)$. The mean age was 27.08 (range 18 to 57 ).

\begin{tabular}{|l|c|c|c|c|c|}
\hline \multirow{2}{*}{ Specialty of students } & \multicolumn{4}{|c|}{ Year No (\%) } & \multirow{2}{*}{ Total } \\
\cline { 2 - 5 } & $1^{\text {st }}$ & $2^{\text {nd }}$ & $3^{\text {rd }}$ & $4^{\text {th }}$ & \\
\hline nursing & $59(20.4)$ & $55(25.3)$ & $42(29.6)$ & $29(37.7)$ & $185(25.5)$ \\
\hline paramedic & $59(20.4)$ & $2(0.9)$ & $1(0.7)$ & $0(0.0)$ & $62(8.6)$ \\
\hline midwifery & $50(17.3)$ & $23(10.6)$ & $31(21.8)$ & $21(27.3)$ & $125(17.2)$ \\
\hline health visitor & $43(14.9)$ & $20(9.2)$ & $19(13.4)$ & $17(22.1)$ & $99(13.7)$ \\
\hline health care management & $49(17.0)$ & $52(24.0)$ & $3(2.1)$ & $0(0.0)$ & $104(14.3)$ \\
\hline social work & $29(10.0)$ & $65(30.0)$ & $46(32.4)$ & $10(12.9)$ & $150(20.7)$ \\
\hline Total & $289(39.9)$ & $217(29.9)$ & $142(19.6)$ & $77(10.6)$ & $725(100)$ \\
\hline
\end{tabular}

Table 1 Year and specialty characteristics of the sample

\section{Choices between CAM and COM on the five levels}

Table 2 shows how students chose between CAM and COM on the five levels of severity of diseases. An example for the interpretation of Table 2: in the first column of the table the four response categories and in the second column the students' choices between the four response categories on the mildest level are shown. Second column involves the students' choices for themselves, their child, parent and partner and the average frequencies of the four response categories in all the four person categories.

Same tendencies were found on each level disease severity: most of the students chose COM+CAM (between 38.8\% and 64.4\%), followed by 'only COM' (between $23.5 \%$ and $28.0 \%$ ), CAM+COM (between $9.7 \%$ and $27.0 \%$ ), and finally, 'only CAM' (between $2.3 \%$ and $6.0 \%$ ).

Students' choices between CAM and COMfor themselves and for their family members

Comparing the frequencies of the four person categories in the four response categories one by one, a significant difference was found on the mildest level in the frequency of 'only CAM' between myself $(8.0 \%, 58 / 725)$ and child $(4.8 \%, 35 / 725)$ $(\mathrm{p}=0.018)$ person categories (Table 2$)$. No other significant differences were found. 


\begin{tabular}{|c|c|c|c|c|c|c|c|c|c|c|c|}
\hline$\stackrel{\vec{D}}{\vec{E}}$ & & $\begin{array}{l}2 \\
2 \\
3 \\
2 \\
3 \\
3\end{array}$ & & $\begin{array}{l}0 \\
\stackrel{2}{c} \\
\delta \\
3\end{array}$ & & $\begin{array}{l}0 \\
3 \\
3 \\
+ \\
\hat{2} \\
3\end{array}$ & & $\begin{array}{l}0 \\
\frac{3}{4} \\
2 \\
2 \\
3\end{array}$ & & 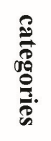 & 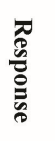 \\
\hline Nun & $\begin{array}{l}\vec{\omega} \\
\infty \\
\infty\end{array}$ & $\stackrel{N}{\mathbb{D}}$ & $\begin{array}{l}\text { 总 } \\
\stackrel{\theta}{0}\end{array}$ & $\begin{array}{l}\vec{\infty} \\
\infty\end{array}$ & $\begin{array}{l}\stackrel{\mathbb{\infty}}{\infty} \\
\underbrace{\infty}\end{array}$ & 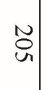 & $\begin{array}{l}\hat{\infty} \\
\stackrel{\theta}{\theta}\end{array}$ & $\stackrel{\sim}{\infty}$ & 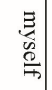 & \multirow{5}{*}{$\frac{z}{\stackrel{2}{\circ}}$} & \multirow{5}{*}{ 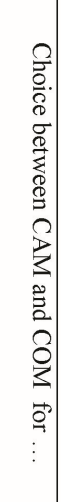 } \\
\hline N & $\begin{array}{l}\underset{\infty}{\infty} \\
\stackrel{\infty}{\infty}\end{array}$ & N్ర & $\begin{array}{l}\mathbb{N} \\
\infty \\
0\end{array}$ & : & 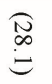 & 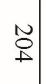 & $\underset{\infty}{\stackrel{f}{\infty}}$ & $\omega_{n}$ & : & & \\
\hline 诘 & $\begin{array}{l}\text { fै } \\
\text { @) }\end{array}$ & $\mathfrak{\Xi}$ & $\begin{array}{l}\text { No } \\
\text { in } \\
\end{array}$ & $\frac{N}{N}$ & $\begin{array}{l}\underset{N}{N} \\
\stackrel{N}{ \pm}\end{array}$ & $\begin{array}{l}\vec{\infty} \\
+\end{array}$ & $\begin{array}{l}\vec{n} \\
\vec{\theta}\end{array}$ & $\omega_{0}$ & 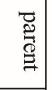 & & \\
\hline \multirow[t]{2}{*}{$\stackrel{N}{N}$} & $\begin{array}{l}\underset{\infty}{\infty} \\
\infty \\
\infty\end{array}$ & $\begin{array}{l}\mathcal{N} \\
\mathbb{N}\end{array}$ & $\begin{array}{l}\mathbb{N} \\
\infty \\
\infty \\
\infty\end{array}$ & $\begin{array}{l}n \\
0\end{array}$ & 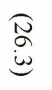 & $\overrightarrow{0}$ & $\begin{array}{l}\hat{n} \\
\ddot{\theta}\end{array}$ & $\stackrel{\omega}{\omega}$ & $\overrightarrow{\underline{a}}$ & & \\
\hline & $\begin{array}{l}\stackrel{\infty}{\infty} \\
\infty \\
\infty\end{array}$ & 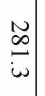 & 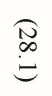 & 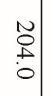 & $\begin{array}{l}\text { N } \\
\text { 仓ै }\end{array}$ & $\begin{array}{l}\vec{b} \\
\circ \\
0\end{array}$ & $\hat{\hat{\theta}}$ & 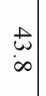 & 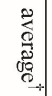 & & \\
\hline Nun & $\begin{array}{l}\underset{+}{N} \\
\text { i }\end{array}$ & $\begin{array}{l}\mathbf{N} \\
\mathbf{\infty}\end{array}$ & 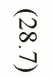 & $\begin{array}{l}N \\
\infty \\
\infty\end{array}$ & $\underbrace{\text { N }}_{\substack{N \\
N}}$ & $\vec{\Xi}$ & $\underset{\omega}{\tilde{\omega}}$ & $N$ & 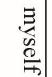 & & \multirow{5}{*}{ 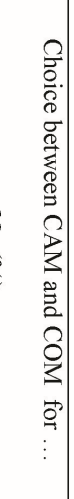 } \\
\hline 偲 & $\underbrace{}_{\substack{\infty \\
\underbrace{}}}$ & $\begin{array}{l}\omega \\
\vec{b}\end{array}$ & $\begin{array}{l}\mathbb{N} \\
\stackrel{\theta}{0}\end{array}$ & : & $\stackrel{\stackrel{N}{=}}{=}$ & $\overrightarrow{\vec{\omega}}$ & $\underset{\underset{v}{\sim}}{\stackrel{i}{N}}$ & $\mathrm{~N}$ & $\stackrel{0}{2}$ & & \\
\hline Nي & $\begin{array}{l}\text { I } \\
\text { E }\end{array}$ & 岕 & 苂 & N & $\stackrel{\text { त् }}{\stackrel{0}{=}}$ & $\vec{a}$ & $\stackrel{\hat{N}}{0}$ & $\vec{\sigma}$ & 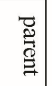 & ż & \\
\hline \multirow[t]{2}{*}{ N } & $\begin{array}{l}\text { f } \\
\stackrel{\vec{b}}{0}\end{array}$ & $\mid$ & 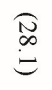 & 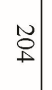 & $\begin{array}{l}\mathbb{N} \\
\stackrel{0}{\oplus}\end{array}$ & $\underset{\infty}{\vec{\infty}}$ & 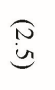 & $\infty$ & $\begin{array}{l}\overline{\underline{u}} \\
\bar{q} \\
\bar{q}\end{array}$ & & \\
\hline & 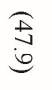 & $\begin{array}{l}\omega \\
\pm \\
\dot{v}\end{array}$ & 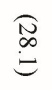 & $\begin{array}{l}0 \\
\stackrel{v}{0} \\
i n\end{array}$ & $\stackrel{\frac{N}{0}}{\stackrel{\theta}{0}}$ & $\begin{array}{l}\vec{u} \\
\stackrel{N}{0} \\
0\end{array}$ & $\begin{array}{l}\hat{0} \\
\dot{\theta}\end{array}$ & $\begin{array}{l}N \\
0 \\
0\end{array}$ & 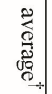 & & \\
\hline N్Nు & $\begin{array}{l}\frac{\partial \pi}{+\infty} \\
\infty \\
\infty\end{array}$ & $\stackrel{\omega}{\omega}$ & $\begin{array}{l}\text { O. } \\
\text { : }\end{array}$ & $\begin{array}{l}N \\
N\end{array}$ & 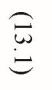 & i & $\widehat{\vec{\omega}}$ & $=$ & 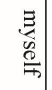 & \multirow{5}{*}{$\frac{z}{\circ}$} & $\stackrel{2}{0}$ \\
\hline Nي & $\begin{array}{l}\text { an } \\
\stackrel{y}{\mathrm{j}}\end{array}$ & $\Xi$ & $\begin{array}{l}\mathbb{0} \\
\text { i্ర }\end{array}$ & $\stackrel{N}{\sim}$ & $\stackrel{\vec{\theta}}{\theta}$ & $\stackrel{\infty}{\infty}$ & $\underset{\overrightarrow{i n}}{(\vec{n}}$ & $=$ & $\stackrel{8}{2}$ & & $\begin{array}{l}\sigma \\
\bar{\sigma} \\
\bar{t} \\
\sigma\end{array}$ \\
\hline Nي & $\underset{\vec{\Xi}}{\stackrel{\vec{\Xi}}{*}}$ & $\stackrel{\vec{t}}{+}$ & $\underset{\vec{\theta}}{\vec{\theta}}$ & $\stackrel{N}{\stackrel{N}{\omega}}$ & $\begin{array}{l}6 \\
\infty \\
\infty\end{array}$ & 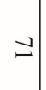 & îv & 0 & 荧 & & 3 \\
\hline 误 & $\begin{array}{l}\text { In } \\
\stackrel{+}{\Perp}\end{array}$ & 함 & 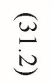 & $\begin{array}{l}N \\
N\end{array}$ & $\begin{array}{l}\vec{\sigma} \\
\dot{0}\end{array}$ & $\checkmark$ & $\underset{\infty}{\stackrel{\infty}{\infty}}$ & $\vec{\omega}$ & 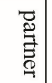 & & $\begin{array}{l}2 \\
\hat{2} \\
3\end{array}$ \\
\hline & $\begin{array}{l}\text { In } \\
\text { iv }\end{array}$ & $\begin{array}{c}0 \\
\infty \\
\infty\end{array}$ & $\stackrel{\oplus}{\stackrel{\theta}{\theta}}$ & $\begin{array}{l}N \\
N \\
+ \\
0\end{array}$ & $\widehat{\overrightarrow{\omega_{0}}}$ & $\begin{array}{l}\infty \\
\stackrel{\infty}{\omega} \\
\stackrel{\omega}{\omega}\end{array}$ & $\widehat{\vec{\omega}}$ & 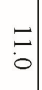 & 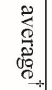 & & $و^{\prime}$ \\
\hline
\end{tabular}

Table 2 Students' choices between CAM and COM for themselves and for their family members on the five levels of severity 


\begin{tabular}{|c|c|c|c|c|c|c|c|c|c|c|c|c|}
\hline $\begin{array}{l}\overrightarrow{0} \\
\vec{d}\end{array}$ & & $\begin{array}{l}2 \\
0 \\
3 \\
+ \\
2 \\
3 \\
3\end{array}$ & & 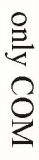 & & $\begin{array}{l}3 \\
3 \\
3 \\
3 \\
3 \\
3\end{array}$ & & $\begin{array}{l}8 \\
2 \\
\Omega \\
z \\
3\end{array}$ & & 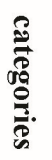 & 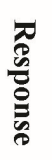 & \\
\hline $\begin{array}{l}\vec{N} \\
\mathbf{u}\end{array}$ & 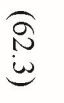 & $\stackrel{\overrightarrow{\mathrm{N}}}{\mathrm{N}}$ & 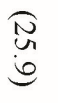 & $\underset{\infty}{\infty}$ & $\stackrel{\substack{0 \\
ن}}{0}$ & ปे & $\stackrel{\overparen{N}}{\stackrel{\theta}{=}}$ & $\vec{v}$ & 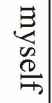 & \multirow{5}{*}{$\frac{z}{\circ}$} & \multirow{5}{*}{ 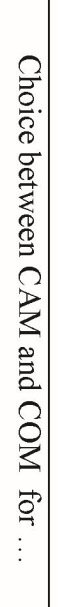 } & \multirow{5}{*}{ 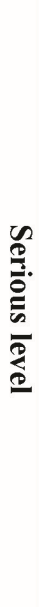 } \\
\hline $\begin{array}{l}\vec{N} \\
\mathbf{u}\end{array}$ & $\begin{array}{l}\widehat{a} \\
\hat{\sigma}\end{array}$ & $\stackrel{\vec{a}}{-}$ & 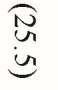 & $\vec{\infty}$ & $\stackrel{6}{\ominus}$ & g & 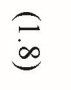 & $\vec{\omega}$ & $\stackrel{?}{\stackrel{2}{2}}$ & & & \\
\hline $\begin{array}{l}\mathbf{N} \\
\mathbf{U}\end{array}$ & 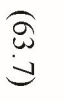 & 吾 & $\begin{array}{l}\underset{\hat{u}}{0} \\
\infty\end{array}$ & $\underset{\infty}{-\infty}$ & $\stackrel{\infty}{\underset{d}{d}}$ & s & $\underset{\infty}{\stackrel{\infty}{\infty}}$ & $\omega$ & 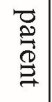 & & & \\
\hline N̦ & 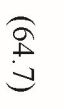 & $\overrightarrow{0}$ & 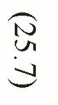 & $\not{\infty}$ & $\underbrace{\infty}$ & 8 & $\stackrel{\vec{\oplus}}{\oplus}$ & $\overrightarrow{0}$ & 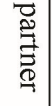 & & & \\
\hline & $\begin{array}{l}\hat{\mathfrak{G}} \\
\hat{\sigma}\end{array}$ & $\stackrel{\vec{a}}{\stackrel{0}{0}}$ & 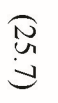 & $\begin{array}{l}\vec{\infty} \\
\text { in }\end{array}$ & $\underbrace{\infty}_{0}$ & $\begin{array}{c}\stackrel{+}{+} \\
\infty\end{array}$ & $\underset{\oplus}{\stackrel{\oplus}{\infty}}$ & $\begin{array}{l}\vec{N} \\
\infty\end{array}$ & 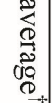 & & & \\
\hline$\vec{N}$ & $\underset{\mathrm{\omega}}{\stackrel{\mathrm{d}}{\mathrm{d}}}$ & $\overrightarrow{8}$ & 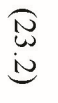 & $\bar{\infty}$ & 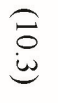 & $\vec{n}$ & $\begin{array}{l}\hat{0} \\
\stackrel{e}{e}\end{array}$ & $N$ & 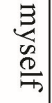 & \multirow{5}{*}{$\frac{z}{\circ}$} & \multirow{5}{*}{$\begin{array}{l}2 \\
3\end{array}$} & \multirow{5}{*}{ 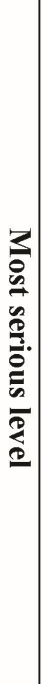 } \\
\hline N̦ & 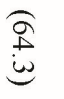 & $\vec{a}$ & $\begin{array}{l}\stackrel{\vec{\omega}}{\omega} \\
\dot{0}\end{array}$ & $\vec{\omega}$ & $\begin{array}{l}6 \\
0 \\
0\end{array}$ & $\mathbf{N}$ & $\stackrel{\overrightarrow{0}}{0}$ & $\vec{\perp}$ & $\stackrel{\varrho}{\bar{E}}$ & & & \\
\hline N & $\begin{array}{l}\hat{\sigma} \\
\stackrel{+}{9}\end{array}$ & $\vec{\infty}$ & 忍 & $\bar{\omega}$ & 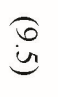 & 8 & $\stackrel{\overparen{N}}{\stackrel{V}{*}}$ & $\vec{u}$ & 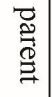 & & & \\
\hline $\begin{array}{l}N \\
\sim\end{array}$ & $\underset{\hat{\sigma}}{\stackrel{\sigma}{u}}$ & $\vec{v}$ & 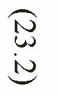 & $\vec{\Phi}$ & $\stackrel{6}{\ominus}$ & হ & $\underbrace{\stackrel{N}{N}}$ & $\vec{\sigma}$ & 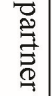 & & & \\
\hline & $\underset{\underbrace{}}{\stackrel{\overbrace{}}{ \pm}}$ & $\begin{array}{l}\vec{a} \\
\vec{a} \\
i\end{array}$ & 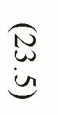 & $\underset{\delta}{\text { in }}$ & $\stackrel{8}{\stackrel{0}{y}}$ & in & $\underbrace{\mathbb{N}}$ & $\begin{array}{c}\vec{a} \\
\infty\end{array}$ & 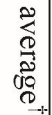 & & & \\
\hline
\end{tabular}

COM: conventional medicine; CAM: complementary and alternative medicine $\dagger$ Frequency of 'myself, child, parent and partner' were added up and divided by 4 .

*Analysis of proportion: $\mathrm{p}<0.05$

Table 2 Students' choices between CAM and COM for themselves and for their family members on the five levels of severity 
Changes in the choice between CAM and COM as the severity of diseases increases

As the level of severity of the diseases increased the average frequency (Figure 2) of 'only CAM' significantly decreased from mildest $(6.0 \%)$ to mild level $(3.0 \%)$ $(p=0.009)$, and it did not change significantly until the most serious level $(2.3 \%)$ $(\mathrm{p}=0.489)$. The average frequency of CAM+COM decreased significantly from mildest $(27.0 \%)$ to mild level $(21.0 \%)(\mathrm{p}=0.009)$ and from mild to moderate level $(11.3 \%)$ $(\mathrm{p}<0.001)$ and it did not change significantly until the most serious level $(9.7 \%)$ $(\mathrm{p}=0.358)$. The average frequency of 'only COM' did not increase significantly from the mildest $(28.1 \%)$ to moderate level $(31.0 \%)(\mathrm{p}=0.274)$, and it significantly decreased up to the most serious level $(23.5 \%)(\mathrm{p}=0.002)$. The average frequency of COM+CAM increased significantly from mildest $(38.8 \%)$ to mild level $(47.9 \%)$ $(\mathrm{p}<0.001)$, and from mild to moderate level $(56.2 \%)(\mathrm{p}=0.002)$ and from moderate to serious level $(63.6 \%)(\mathrm{p}=0.005)$ and it did not change until the most serious level $(64.5 \%)(p=0.772)$.

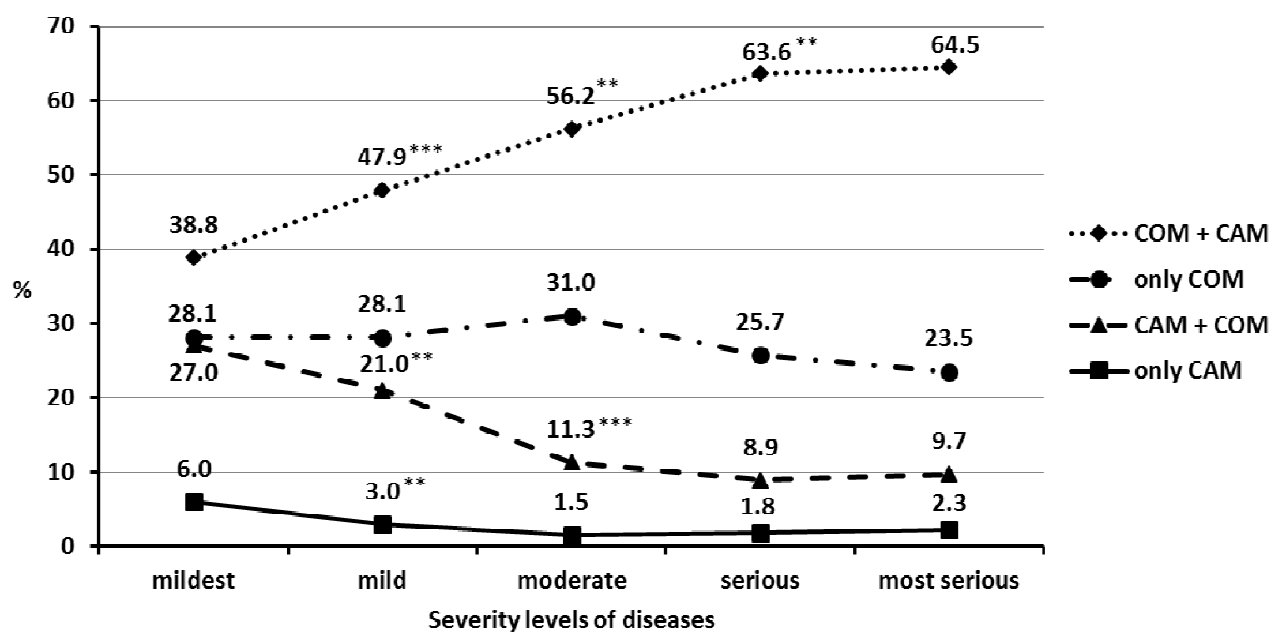

COM: conventional medicine; CAM: complementary and alternative medicine Analysis of proportion

$* \mathrm{p}<0.05 ; * \mathrm{*}<0.01 ; * * * \mathrm{p}<0.001$

Figure 2 Changes in the average frequencies of the four response categories as the severity of diseases increases.

Gender differences in the choice between CAM and COM on the five levels of severity

Table 3 shows how male and female students chose between CAM and COM on the five levels of severity of diseases. An example for the interpretation of Table 3: in the first column of the table the four response categories and in the second column the students' choices between the four response categories on the mildest level are shown. 
Second column involves the average frequencies of the four response categories for males and females.

A significant difference was found on the mildest level (Table 3) in the average frequency of 'only COM' between males $(37.6 \%, 35 / 93)$ and females $(26.7 \%$, $169 / 632)(\mathrm{p}=0.040)$. On mild and moderate levels gender differences were not found. On serious level the average frequency between males and females was significantly different in 'only COM' $(37.9 \%, 35.3 / 93$ vs. $23.9 \%, 151.3 / 632, \mathrm{p}=0.006)$ and in COM+CAM (50.8\%, 47.3/93 vs. $65.4 \%, 413.8 / 632, \mathrm{p}=0.009)$. Similarly, on the most serious level the average frequency between males and females was significantly different in 'only COM' $(38.2 \%, 35.5 / 93$ vs. $21.4 \%, 135 / 632, \mathrm{p}=0.001)$, and in $\mathrm{COM}+\mathrm{CAM}(50.8 \%, 47.3 / 93$ vs. $66.4 \%, 420 / 632, \mathrm{p}=0.005)$.

\begin{tabular}{|c|c|c|c|c|c|c|c|c|c|c|}
\hline \multirow{4}{*}{$\begin{array}{c}\text { Response cat } \\
\text { egories }\end{array}$} & \multicolumn{10}{|c|}{ Average frequency ${ }^{\dagger}$ No $(\%)$} \\
\hline & \multicolumn{2}{|c|}{ Mildest level } & \multicolumn{2}{|c|}{ Mild level } & \multicolumn{2}{|c|}{ Moderate level } & \multicolumn{2}{|c|}{$\begin{array}{c}\text { Serious } \\
\text { level }\end{array}$} & \multicolumn{2}{|c|}{$\begin{array}{c}\text { Most serious } \\
\text { level }\end{array}$} \\
\hline & male & fe & male & female & male & female & male & female & male & female \\
\hline & $\mathrm{N}=93$ & $\mathrm{~N}=632$ & $\mathrm{~N}=93$ & $\mathrm{~N}=632$ & $\mathrm{~N}=93$ & $\mathrm{~N}=632$ & $\mathrm{~N}=93$ & $\mathrm{~N}=632$ & $\mathrm{~N}=93$ & $\mathrm{~N}=632$ \\
\hline onl & \begin{tabular}{|c|}
2.5 \\
$(2.7)$
\end{tabular} & \begin{tabular}{|l|}
41.3 \\
$(6.6)$
\end{tabular} & $\begin{array}{c}2.8 \\
(3.0)\end{array}$ & $\begin{array}{l}19.3 \\
(3.0)\end{array}$ & $\begin{array}{c}1 \\
(1.1)\end{array}$ & $\begin{array}{c}10 \\
(1.6)\end{array}$ & $\begin{array}{c}1 \\
(1.1)\end{array}$ & $\begin{array}{l}11.8 \\
(1.9)\end{array}$ & $\begin{array}{c}1 \\
(1.1)\end{array}$ & $\begin{array}{l}15.8 \\
(2.5)\end{array}$ \\
\hline $\mathrm{CA}$ & $\begin{array}{c}24 \\
(25.8)\end{array}$ & $\begin{array}{c}172 \\
(27.2)\end{array}$ & $\begin{array}{c}18 \\
(19.3)\end{array}$ & $\begin{array}{c}134 \\
(21.2)\end{array}$ & $\begin{array}{c}10.8 \\
(11.6)\end{array}$ & $\begin{array}{c}71.5 \\
(11.3)\end{array}$ & $\begin{array}{c}9.5 \\
(10.2)\end{array}$ & $\begin{array}{l}55.3 \\
(8.8)\end{array}$ & $\begin{array}{c}9.3 \\
(10.0)\end{array}$ & $\begin{array}{l}61.3 \\
(9.7)\end{array}$ \\
\hline on & $\begin{array}{c}35^{*} \\
(37.6)\end{array}$ & $\begin{array}{c}169 \\
(26.7)\end{array}$ & $\begin{array}{c}33 \\
(35.5)\end{array}$ & $\begin{array}{l}170.5 \\
(27.0)\end{array}$ & $\begin{array}{c}35.8 \\
(38.4)\end{array}$ & $\begin{array}{l}188.3 \\
(29.8)\end{array}$ & $\begin{array}{l}35.3^{*} \\
(37.9)\end{array}$ & $\begin{array}{l}151.3 \\
(23.9)\end{array}$ & $\begin{array}{l}35.5^{*} \\
(38.1)\end{array}$ & $\begin{array}{c}135 \\
(21.3)\end{array}$ \\
\hline $\mathrm{COM}$ & $\begin{array}{l}31.5 \\
(33.9)\end{array}$ & $\begin{array}{l}249.8 \\
(39.5)\end{array}$ & \begin{tabular}{|c|}
39.3 \\
$(42.2)$
\end{tabular} & $\begin{array}{l}308.3 \\
(48.8)\end{array}$ & $\begin{array}{c}45.5 \\
(48.9)\end{array}$ & $\begin{array}{l}362.3 \\
(57.3)\end{array}$ & $\begin{array}{l}47.3^{*} \\
(50.8)\end{array}$ & $\begin{array}{l}413.8 \\
(65.4)\end{array}$ & $\begin{array}{l}47.3^{*} \\
(50.8)\end{array}$ & $\begin{array}{c}420 \\
(66.4)\end{array}$ \\
\hline
\end{tabular}

$\mathrm{N}=725$

COM: conventional medicine; CAM: complementary and alternative medicine

$\dagger$ Frequency of 'myself, child, parent and partner' were added up and divided by 4 for males and females.

*Analysis of proportion: $\mathrm{p}<0.05$

Table 3 Gender differences in the average frequencies of the four response categories on the five levels of severity.

Gender difference in the choice between CAM and COM as the severity of the diseases increases

As the severity of the diseases increased (Figure 3), the average frequency among females in 'only CAM' significantly decreased from the mildest $(6.6 \%)$ to mild level $(3.0 \%, p=0.006)$ and it did not change significantly until the most serious level $(2.5 \%)(\mathrm{p}=0.669)$. As for males the average frequency of 'only CAM' did not significantly change from the mildest $(2.7 \%)$ to the most serious level $(1.1 \%)$ (p value could not be counted due to low sample number). The average frequency in $\mathrm{CAM}+\mathrm{COM}$ among females significantly decreased from the mildest $(27.2 \%)$ to 
mild level $(21.2 \%)(\mathrm{p}=0.015)$, and from mild to moderate level $(11.3 \%)(\mathrm{p}<0.001)$, but it did not change significantly until the most serious level $(9.7 \%)(p=0.394)$. As for males the average frequency in $\mathrm{CAM}+\mathrm{COM}$ significantly decreased from the mildest $(25.8 \%)$ to moderate level $(11.6 \%)(\mathrm{p}=0.022)$, but it did not change significantly until the most serious level $(10.0 \%)(\mathrm{p}=0.906)$. The average frequency in 'only COM' among females did not increase significantly from the mildest $(26.7 \%)$ until to moderate level $(29.8 \%)(\mathrm{p}=0.253)$, and it significantly decreased from moderate to serious level $(23.9 \%)(\mathrm{p}=0.022)$, and it did not change significantly to the most serious level $(21.4 \%)(\mathrm{p}=0.303)$. As for males the average frequency of only COM did not significantly change from the mildest $(37.6 \%)$ to the most serious level $(38.2 \%)(\mathrm{p}=1.0)$, it was almost $40 \%$ on each level. The average frequency in COM+CAM among females significantly increased from the mildest $(39.5 \%)$ to mild level $(48.8 \%)(p=0.001)$, and from mild to moderate level $(57.3 \%)(p=0.003)$, and from moderately to serious level $(65.4 \%)(\mathrm{p}=0.003)$, and it did not change significantly to the most serious level $(66.4 \%)(p=0.758)$. As for males the average frequency significantly increased in COM+CAM from the mildest (33.9\%) to serious level $(50.8 \%)(\mathrm{p}=0.003)$, and it did not change significantly until the most serious level $(50.8 \%)(\mathrm{p}=0.907)$.

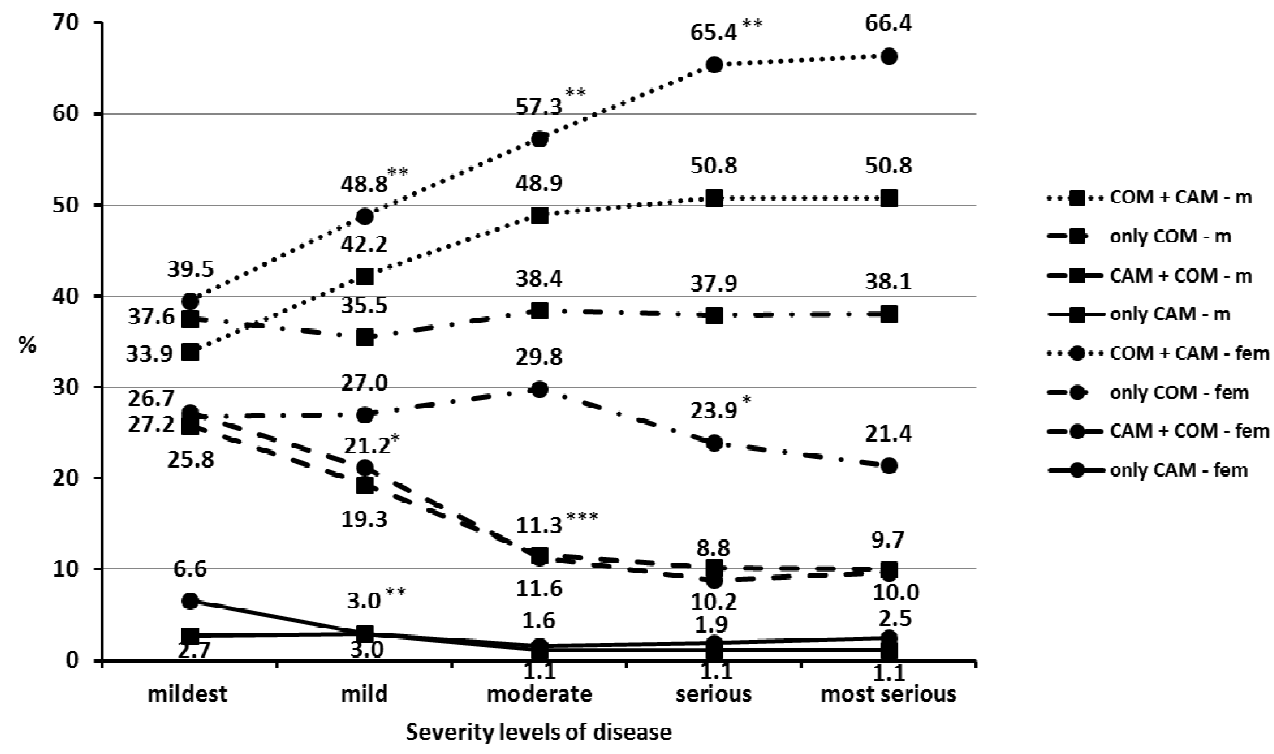

COM: conventional medicine; CAM: complementary and alternative medicine; m: males; fem: females

$* \mathrm{p}<0.05 ; * * \mathrm{p}<0.01 ; * * * \mathrm{p}<0.001$

Figure 3 Gender differences in the average frequencies of the four response categories as the severity of the diseases increases 


\section{Discussion}

Although people in European countries are growing up in a cultural environment where the norm for treating diseases is conventional therapy, over the last decades the popularity and use of CAM methods (e.g. natural products) has increased both among the general population and patients with chronic diseases (Sibbritt, Davidson et al. 2015, Büssing, Ostermann, et al. 2011, Fox, Coughlan et al. 2010, Ceylan, Azal et al. 2009, Barnes, Bloom and Nahin 2008, Chang, Wallis et al. 2007, Molassiotis, Fernadez-Ortega et al. 2005b). Even our results, which found that on each five level most of the respondents chose COM+CAM followed by 'only COM' and CAM+COM, with only a minority of them choosing 'only CAM', correspond to these norms and reflect the predictions.

Significant gender differences were found in choosing between CAM and COM. The average frequency of 'only COM' was significantly higher among males than females on each level and the difference was significant on the mildest, serious and the most serious levels of diseases (the absolute differences were 10-17\%). The average frequency of $\mathrm{COM}+\mathrm{CAM}$ was higher among females than males on each level and the difference was significant on serious and the most serious levels of diseases (the absolute differences were 15\%). These results, supported by the data of previous studies, show that females have a more positive attitude towards CAM and they are more open toward CAM than males (Akan, Izbirak and Kaspar 2012, Awad, Al-Ajmi and Waheedi 2012, Greenfield, Brown et al. 2006).

As the severity of diseases increased the average frequency of $\mathrm{COM}+\mathrm{CAM}$ also increased from mildest to the most serious level by $26 \%$ and on the most serious level of diseases almost two thirds of students chose this option $(64.5 \%)$. The average frequency decreased from the mildest to the most serious level by nearly one third in $\mathrm{CAM}+\mathrm{COM}$ (from $27 \%$ to $9.7 \%$ ), as well as in only CAM (from $6.0 \%$ to $2.3 \%$ ), as well as in 'only COM' it decreased from moderate to the most serious level (from $31.0 \%$ to $23.5 \%$ ). These results clearly show that one part of the students changed their choices from $\mathrm{CAM}+\mathrm{COM}$ and from 'only COM' to COM+CAM.

Regarding gender differences, as the severity of diseases increased, significant decreases were observed among females in the average frequency of 'only CAM' (from the mildest to mild level), $\mathrm{CAM}+\mathrm{COM}$ (from the mildest to mild and from mild to moderate level), and 'only COM' (from moderate to serious level). In line with this, among females the average frequency of COM+CAM significantly increased from level to level up to the most serious level. Among males the average frequency of $\mathrm{CAM}+\mathrm{COM}$ significantly decreased (from the mildest to moderate level) and it significantly increased in COM+CAM (from the mildest to serious level). Thus, based upon the data we conclude that one part of both female and male students changed their choices between CAM and COM as follows. One part of both males and females shifted on the mild level while the other part shifted on the moderate level from $\mathrm{CAM}+\mathrm{COM}$ to $\mathrm{COM}+\mathrm{CAM}$. The third part of the females probably shifted their choices on the serious level from 'only COM' to COM+CAM. The fourth part of the females shifted on the mild level from only CAM to other categories, but it is unclear to which one. 
These shifts are supposed to be influenced by students' knowledge and beliefs about conventional medicine and CAM, by their previous use of and experience with CAM, social and cultural norms, as well as professional socialization (Lorenc, Blair and Robinson et al 2014, Weeks, Balneaves et al. 2014). Our previous studies have found that the attitudes of health care students towards CAM were positive at our faculty, and they had relatively high level of knowledge and personal use of CAM (Sárváry, Demcsák et al. 2016).

Moreover, these results also support students' belief that besides conventional medicine CAM is an important element of medical treatment on each severity level of diseases. Our previous results also found that health care students were convinced that the integration of CAM in the curricula is needed (Sárváry, Demcsák et al. 2016), since that could help to improve the holistic approach of treating diseases (Sárváry, Demcsák et al. 2016, Çamurdan and Gül 2013, Akan, Izbirak and Kaspar 2012, Awad, Al-Ajmi and Waheedi 2012). In future studies further analyses are needed to detect the patterns of the choice between CAM and conventional medicine as the severity of diseases increases.

Students' choices for themselves and for their family members between CAM and COM were similar, except on the mildest level, where significantly more students chose the 'only CAM' for themselves than for their children. Our results can be explained by cognitive consistency theories. According to these theories people seek consistency in their thinking, so they try to avoid contradictions, because it results in an uncomfortable, tense state (dissonance) (Smith and Mackie 2007). Our results suggest that students (can) think on each 5 level of diseases that "what is good for me is also good for my family members'. By thinking so they can avoid the phenomenon of cognitive dissonance (Haddock and Maio 2012, Smith and Mackie 2007). In further studies other 'person-categories' (e.g. patient) should be involved to study the difference in the choice between CAM and COM.

Our study had some limitations. First, using self-reported questionnaire answers could be influenced by social desirability therefore our results may not reflect students' real choices between CAM and conventional medicine. Second, we carried the study among students, therefore the results can only be generalised to this population. In the future we should repeat the study with a sample from a population of general adults. Third, although our sample was representative with respect to the proportion of gender at the Faculty of Health (at our faculty the majority of students are female), further investigation is needed to determine whether gender differences would be found in a population with a more balanced male-female representation. Moreover, students had only one chance to complete the questionnaire and this may have contributed to the $44 \%$ response rate. Finally, in the case of 'child' category, answers of students without a child may not reflect their real choice due to lack of experience in caring for a child.

\section{Conclusion}

On each level of severity most of the students chose 'primarily COM, secondly CAM', followed by 'only COM', 'primarily CAM, secondly COM', and finally, 'only CAM'. 
Our results supported that conventional medicine is dominant in medical treatment in the European culture, but CAM is also regarded as an important part of the healing process. Students' choices between CAM and conventional medicine were found to be consistent for themselves and for their family members. Significantly more males than females chose 'only COM' on the mildest, serious and the most serious levels and significantly more females than males chose 'primarily COM and secondly CAM' on serious and the most serious levels of disease. Our results supported the position that females are more open towards CAM than males. The more serious the disease was, the response rate in 'primarily COM and secondly CAM' increased while it decreased in 'primarily CAM and secondly COM', in 'only CAM' and in 'only COM'. As the severity of disease increased one part of students changed their choices between CAM and COM. The changes in the choice between CAM and conventional medicine - and their gender difference- are needed to be investigated in further studies. It is possible to find some typical patterns in the choices between CAM and conventional medicine as the severity of diseases increases.

\section{Acknowledgements}

We are thankful to the following colleagues of the Faculty of Health for their assistance in this study: Éva Huszti PhD, Katalin Papp PhD, and Ágnes Tilki, and also to Professor Thomas R. Lawson PhD University of Louisville, Kentucky, US.

\section{Footnotes}

${ }^{1}$ Health visitors are preventive professionals, who generally care for children until 18 years. They either work in districts with pediatric family doctors or in schools.

\section{Conflict of interest statement}

None.

1. Akan H, Izbirak G, Kaspar EC, Kaya CA, Aydin S, Demircan N, Bucaktepe PG, Özer C, Sahin HA, Hayran O (2012): Knowledge and attitudes towards complementary and alternative medicine among medical students in Turkey. BMC Complement Altern Med. 2012;12: 115.

2. Akinci AC, Zengin N, Yildiz H, Sener E, Gunaydin B. (2011): The complementary and alternative medicine use among asthma and chronic obstructive pulmonary disease patients in the southern region of Turkey. Int $\mathrm{J}$ Nurs Pract 2011;17571-82, doi:10.1111/j.1440-172X.2011.01976., 
3. Akyol AD, Oz B. (2011): The use of complementary and alternative medicine by patients with cancer: in Turkey. Complement Ther Clin Pract 2011;17: 230-4, doi:10.1016/j.ctcp. 2010.12.003.

4. Antal Z, Szántó Zs. (1992): A természetgyógyászat és az orvostudomány konfliktusa. Leltár. MTA Társadalmi Konfliktusok Kutató Központjának kiadványa. Budapest.

5. Awad AI, Al-Ajmi S, Waheedi MA. (2012): Knowledge, Perceptions and Attitudes toward Complementary and Alternative therapies among Kuwaiti Medical and Pharmacy Students. Med Princ Pract 2012;21: 350-354.

6. Barnes PM, Bloom B, Nahin RL. (2008): Complementary and alternative medicine use among adults and children: United States, 2007. Natl Health Stat Report. 2008; 12: 1-23.

7. Buda L. (2003): Az alternatív medicina tudományos orvosláshoz való viszonyának, társadalmi-lélektani, egészség-szociológiai hátterének elemzése és az egészségügyi ellátásban betöltött szerepének complex empirikus vizsgálata PhD értekezés. Pécsi Tudományegyetem Általános Orvostudományi Kar.

8. Büssing A, Ostermann T, Heusser P, Matthiessen PF. (2011): Usage of alternative medical systems, acupuncture, homeopathy and anthroposophic medicine, by older German adults. Zhong Xi Yi Jie He Xue Bao. 2011;9: 847-56.

9. Çamurdan Ç, Gül A. (2013): Complementary and alternative medicine use among undergraduate nursing \& midwifery students in Turkey. Nurse Educ Pract 2013;13: 350-4.

10. Ceylan S, Azal O, Taşlipinar A, Türker T, Açikel CH, Gulec M. (2009): Complementary and alternative medicine use among Turkish diabetes patients. Complement Ther Med 2009; 17:78-83.

11. Chang HY, Chang HL. (2015): A review of nurses' knowledge, attitudes, and ability to communicate the risks and benefits of complementary and alternative medicine. J Clin Nurs. 2015;24: 1466-78.

12. Chang HY, Wallis M, Tiralongo E. (2007): Use of complementary and alternative medicine among people living with diabetes: literature review. J Adv Nurs 2007;58: 307-19.

13. Crowe S, Fitzpatrick G, Jamaluddin MF. (2002): Use of herbal medicines in ambulatory surgical patients. Anaesthesia. 2002;57: 183-208.

14. Dhanoa A, Yong TL, Yeap SJ, Lee IS, Singh VA. (2014): Complementary and alternative medicine use amongst Malaysian orthopaedic oncology patients. BMC Complement Altern Med. 2014;14: 404. doi:10.1186/1472-6882-14-404

15. Európai Lakossági Egészségfelmérés (ELEF) - Magyarország, 2009. Öszefoglaló eredmények. [National Population Health Survey. Research report]. Központi Statisztikai Hivatal 2011, Budapest.

16. Fountouki A, Theofanidis D. (2009): Nurses' attitudes towards complementary therapies. Health Sci J 2009;3: 149-157.

17. Fox P, Coughlan B, Butler M, Kelleher C. (2010): Complementary alternative medicine (CAM) use in Ireland: a secondary analysis of SLAN data. Complement Ther Med. 2010;18: 95-103.

18. Greenfield SM, Brown R, Dawlatly SL, Reynold JA, Roberts S, Dawlatly RJ. (2006): Gender differences among medical students in attitudes to learning about 
complementary and alternative medicine. Complement Therap Med 2006;14: 207-212.

19. Haddock G, Maio GR. Attitudes. In: Hewstone M, Stroebe W, Jonas K [eds.] (2012): An introduction to social psychology, 5th ed. Glasgow: BPS Blackwell. 171-200.

20. Harris PE, Cooper KL, Relton C, Thomas KJ. (2012): Prevalence of complementary and alternative medicine (CAM) use by the general population: a systematic review and update. Int J Clin Pract. 2012;66: 924-39. doi:10.1111/j.17421241.2012.02945.

21. Horneber M, Bueschel G, Dennert G, Less D, Ritter E, Zwahlen M. (2012): How many cancer patients use complementary and alternative medicine: A systematic review and metaanalysis. Integr Cancer Ther. 2012;1: 187-203.

22. John GM, Hershman DL, Falci L, Shi Z, Tsai WY, Greenlee H, (2016): Complementary and alternative medicine use among US cancer survivors. J Cancer Surviv. 2016 Oct;10(5):850-64. doi: 10.1007/s11764-016-0530-y. Epub 2016 Feb 26.

23. Lorenc A, Blair M, Robinson N. (2014): Personal and professional influences on practitioners' attitudes to traditional and complementary approaches to health in the UK. Journal of Traditional Chinese Medical Sciences 2014; 1:148-155.

24. Mandreker B. (2015): Complementary and alternative medicine usage among cardiac patients: a descriptive study. BMC Complement Altern Med. 2015;15: 100.

25. Molassiotis A, Fernandez-Ortega P, Pud D, Ozden G, Platin N, Hummerston S, et al. (2005a): Complementary and alternative medicine use in colorectal cancer patients in seven European countries. Complement Ther Med 2005a;13: 251-7.)

26. Molassiotis A, Fernadez-Ortega P, Pud D, Ozden G, Scott JA, Panteli V, Margulies A, Browall M, Magri M, Selvekerova S, Madsen E, Milovics L, Bruyns I, Gudmundsdottir G, Hummerston S, Ahmad AM, Platin N, Kearney N, Patiraki E. (2005b): Use of complementary and alternative medicine in cancer patients: a European survey. Ann Oncol. 2005b;16: 655-63.

27. Ni H, Simile C, Hardy AM. (2002): Utilization of complementary and alternative medicine by United States adults: results from the 1999 national health interview survey. Med Care. 2002;40(4):353-8.

28. Norred CL. (2002): Complementary and alternative medicine use by surgical patients. ORN J. 2002;7(6):1013-21.

29. Norred CL, Zamudio S, Palmer SK. (2000): Use of complementary and alternative medicines by surgical patients. AANA J. 2000; 68:13-8.

30. Reid R, Steel A, Wardle J, Trubody A, Adams J. (2016): Complementary medicine use by the Australian population: a critical mixed studies systematic review of utilisation, perceptions and factors associated with use. BMC Complement Altern Med. 2016;16: 176. doi:10.1186/s12906-016-1143-8.

31. Sárváry A, Demcsák HL, Radó S, Takács P, Sárváry A. (2016): Students' nurses, midwives and health visitors' attitudes, knowledge, and experience of complementary and alternative medicine in Hungary. European Journal of Integrative Medicine, 2016, 8, (4): 552-559. 
32. Schieman C, Rudmik LR, Dixon E, Sutherland F, Bathe OF. (2009): Complementary and alternative medicine use among general surgery, hepatobiliary surgery and surgical oncology patients. Can J Surg. 2009;52(5): 422-6.

33. Shorofi SA, Arbon P. (2010): Nurses' knowledge, attitudes, and professional use of complementary and alternative medicine (CAM): a survey at five metropolitan hospitals in Adelaide. Complement Ther Clin Pract 2010;16: 229-34.

34. Sibbritt D, Davidson P, DiGiacomo M, Newton P, Adams J. (2015): Use of Complementary and Alternative Medicine in Women With Heart Disease, Hypertension and Diabetes (from the Australian Longitudinal Study on Women's Health). Am J Cardiol 2015;115: 1691-5.

35. Smith ER, Mackie DM.(2007): Attitudes and Behavior. In: Smith ER, Mackie DM. Social Psychology, 3rd ed. New York: Taylor and Francis Group, 2007: 269-306.

36. Soós SÁ, Jeszenői N, Darvas K, Harsányi L. (2015): Herbal medicine use by surgery patients in Hungary: a descriptive study. BMC Complement Altern Med 2015; 15: 358. doi: 10.1186/s12906-015-0890-2.

37. Sparber A, Wootton JC. (2001): Surveys of complementary and alternative medicine: Part II. Use of alternative and complementary cancer therapies. J Altern Complement Med 2001; 7: 281-7.

38. Statistical Data Analysis (R); Test of Equal or Given Proportions. Available at: https://stat.ethz.ch/R-manual/R-devel/library/stats/html/prop.test.html. Accessed: 12 Nov 2016.

39. Trail-Mahan T, Mao CL, Bawel-Brinkley K. (2013): Complementary and Alternative Medicine: Nurses' Attitudes and Knowledge. Pain Manag Nurs 2013;14: 277-286.

40. Wang SM, Peloquin C, Kain ZN. (2002): Attitudes of Patients Undergoing Surgery Toward Alternative Medical Treatment. J Altern Complement Med. 2002;8: 351-56.

41. Wootton JC, Sparber A. (2001): Surveys of complementary and alternative medicine: Part III. Use of alternative and complementary therapies for HIV/AIDS.J Altern Complement Med 2001;7: 371-7

42. Weeks L, Balneaves LG, Paterson C, Verhoef M. (2014): Decision-making about complementary and alternative medicine by cancer patients: integrative literature review. Open Med 2014;8: 54-66. eCollection 2014. 